\title{
Research on Development Track of Computer Music and the Influences on Musical Creation
}

\author{
Sui Wu \\ Hunan City University, \\ Yiyang,Hunan,413000 China
}

\begin{abstract}
In this paper, we conduct research on the development track of the computer music and the influences on musical creation. With the advent of the new era of digital music, new music through also started the personal computer revolution. We only have one computer can onto the road of their own music and creative hobbies. Computer music software for all lovers of music, music learners and provides a broad space of infinite music workers: it more into music lovers to indulge in all sorts of wonderful music; It makes the music learners to learn all kinds of music knowledge more efficiently; It makes music workers can follow one's inclinations the use of a variety of voice quality and creative skills, produce more wonderful music works. To further development of the creation procedures, we propose the novel paradigm that will hold special meaning.
\end{abstract}

Keywords- Development Track, Computer Music, Musical Creation, Basic Influences.

\section{Introduction}

The narrow sense of computer music refers to the kind of data by computer calculation processing functions provided by the data as the sound of music material and the main parameters in the music source of music types. So far, the academic consensus is: the use of computer music is just a tool, not a music style. For a wide range and is still in its early stage of development of computer music is concerned and this judgment is surely right. But it is undeniable that musicians in the special use computer tools to make music, has been applied and is continuing to develop many different with the traditional music and even opposite ideas, concepts and methods [1-3].

In fact, in the human music activities there is also another with participation of subjective factors other than the generation of music parameters. We can call this the "interactive" creation. On the way of interaction, and randomly generated and calculus generated, generated by hand and basic machine automatically generated a variety of the ways. Any computer music system is inseparable from the support of software, it is the soul of the whole system, phyletic and various, countless. According to their respective functions can be roughly divided into the following categories. (1) Music printing software. There have prepared a lot of music symbols, such as the attachment, gradually strong, weak, fermata, skipping and repeatedly, and even professional guitar spectrum or drum symbol, also allows user to make its own symbol, just connected to the printer and we can get nice music. (2) Automatic accompaniment software. As the waltz in classical and Haydn, Mozart's music style, or modern style of jazz, pop, etc. have been software editor presets. Users just enter chords and choose a style can have several parts of accompaniment music, melody and can input, form a complete works of music. (3) Sequence of software. Played its role is to put the MIDI keyboard or the mouse to click the notes recorded in the form of digital signals and displayed in the form of music or digital, for users to edit any processing can also be edited music in the form of computer files stored on disk or CD.

General composition is to let every learning music, to form into the music ontology language, 
and degree can be divided into depth, but strengthen the purpose of music learning is consistent, this is social music education is an important part of is also develop new composers social foundation and the important role of the audience. In the music creation we should try to overcome the two extreme ways of thinking. (1) Emphasis on individual subjective feeling, only to feel instead of the actual writing process, ignore the composing system of basic training; By perceptual understanding instead of rational thinking, and thus lack the necessary performance techniques and structure design, written works of music from the stereo to expression is straightforward and shallow. (2) A notation on the surface of the form of the "new" represents a sound, but cannot replace the author real art originality. Only in the sense of music performance and technique means appropriate to the consistent, the unity of time, is it possible to achieve a decent music works of art taste, and the corresponding mental state.

To enhance the traditional computer music paradigm, in this paper, we conduct research on the development track of computer music and the influences on the musical creation. Music creation is different from other art: the unity of the opposites of time and space and the unity of opposites of the representational and abstract. The pair of the characteristic extremely the contradictions, constituted the particularity of the music creation can use dialectical point of view, and creatively recognize and deal with them that is the test of the composer [4-5].

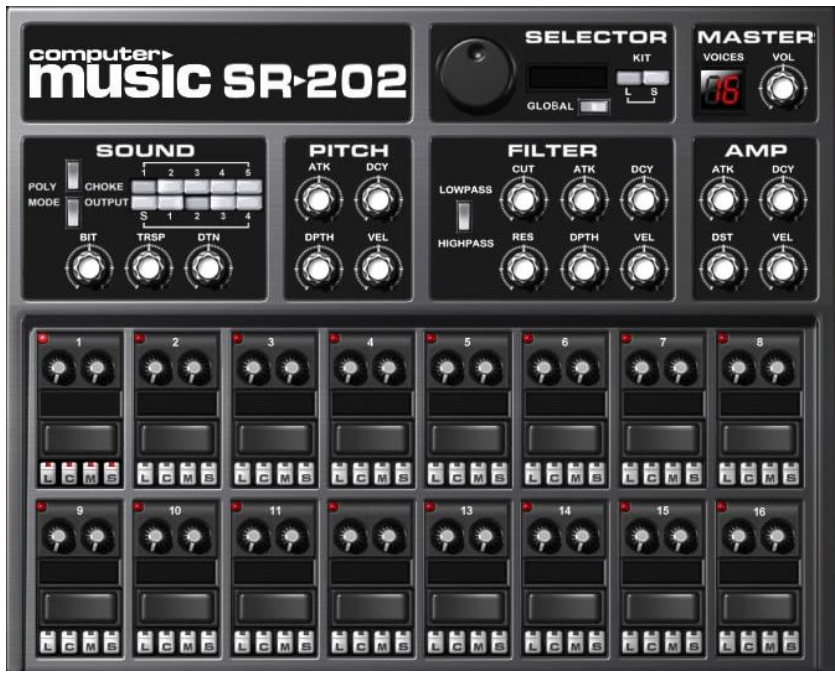

Figure 1. The Illustartion of the Sample Computer Music Platform

\section{The Proposed Perspective}

The Music Aesthetics. Aesthetics is a social science, and music aesthetics is a branch of general aesthetic, one part. It studies on the reality, especially for art aesthetic relations. It to solve human characteristics, origin and development of aesthetic consciousness, and its various reflected in art but also on the essence of art and its law of the development, the relationship between art and reality, the essence of beauty, beauty standards, and the other issues.
In a way between Chinese and western aesthetic culture is also has a lot in common. But in the category of art activities and the specific form, it showed considerable differences between Chinese and western. So far, very few people to compare Chinese and western music culture, our focus is not in China, that also is not the classification of the western music esthetics thought, but through the comparison of music aesthetics features more in-depth to understand the cultural essence of Chinese traditional music, to help us to better establish the Chinese nation 
spirit culture connotation, to better grasp the value orientation of national culture [6].

The basic connotation of music aesthetics could be generally summarized as the follows. (1) Music perception needs aesthetic subject subjective participation at the same time. Music perception is also inseparable from the human subjective initiative and the objective object of music under the premise of existence, human should play their subjective evaluation and judgment. (2) Music as an important part of music aesthetic perception, it produces must also and the aesthetic object is the premise of the existence of music art. (3) Aesthetic evaluation is the important link of music aesthetics, the music is good or bad to make the right evaluation will have certain aesthetic standards to some extent people's identity to some music and the standard which is the basis of the disgust or have in common. (4) The different social historical period, people of different nationalities, different class status evaluation and judgment of the same music is different, this is because the music evaluation under the restriction of the local concrete historical condition at that time.

People ever green main analysis and research of music aesthetics thought, pay more attention to the understanding of the text itself, to a certain extent, played down the green the source and formation of the main music esthetics thought, the green lord accepted music esthetics thought and thought, the relationship between and how he will accept and use the aesthetic thought of subjective factors, thus impedes our correct understanding of its essence of music aesthetics ideology and judging.

Music is the sound performance means, to hearing the voice of the object of art, or is called the auditory art. The sound of music art, mainly by people according to the aesthetic principle, processing created, and consists of a series of music according to the different pitch an orderly constitute an organic whole, the music in the music system. In music aesthetics, music system, based on its unique beauty of rhythm law constitutes a form of the music art language beauty. Its performance way, can directly express people's feelings, also can adopt indirect way, borrow music language to describe social life scenes or basic natural scenery to express people's feelings and will, express thought and experience of life and life for people to create a kind of unique artistic conception, music art creates unique music artistic image. In many ethnic groups in China's traditional culture, music practical significance and aesthetic significance and western classical music and even all professional music is different. Pitch is identified and as a result of the actual music decree, the rhythm is more elastic, individual nation "harmonic interval" no big minor function, so there is no system of harmony and polyphony developed in the west, it is different from western aesthetic habits and aesthetic tastes.

The Musical Creation Procedures. Of rational thinking in music creation, tend to emphasize creation theory and creation rule, composition law, the dominant role in the creation of easy to ignore individual incited, creative mood, emotion and creative intuition and other perceptual driven, inertial force in the creation. Compared with the rational thinking, the creation of the perceptual thinking is sometimes to play to the artistic expression of the intangible obstacles, invisible killer, affecting the quality of the artistic creation and in the creation of trying to get rid of the creation of emotional factors, in an attempt to manifest itself in the center position in the music creation [7].

The digital age, go to the public music creation is unprecedented, digital music, with a new attitude to expand traditional music creation, performance and the connotation and form of communication. In the digital age, everyone can become the "artist". The characteristics of the digital age, that is, all the works of music can be Shared with the quickest speed, a lot of music works are spread, many people depend on their hobbies and try to borrow online and digital own music dream, traditional composer's supreme 
status is being challenged. The detailed musical creation procedures are shown beow.

\section{Music Creation Processes}

Composition, interpretation, improvisation, and arranging are four key methods of musical creation processes.

* Composition involves planning out the design of a work prior to its performance.

* Interpretation is the process through which performers or listeners make an existing composition 'their own' through the experience of performance or listening.

Figure 2. The Detailed Musical Creation Procedures

From the above analysis, the reason and perceptual creation, music creation in the actual creation may be divided into two distinct forms of thinking and the creation of the two competing methods. But analyze the successful creation of the whole music works as you can see, the perceptual thinking and rational thinking are used in combination with each other to the creation of the music and they are interdependent, depend on each other, the development of the complementary, make the music work innovation, which fully reflects the beauty and function of the music. Music creation inspiration, and was absorbed in the thinking, and sometimes inadvertently, from time to time in the sleepy. But the generation of inspiration is no mystery it is people through long-term practice with the experience we summarize suddenly clarified in process of rational thinking is practical experience and knowledge accumulation to a certain degree of intellectual understanding. People will think, sensibility is the power of creation, perceptual represents a creative desire and passion of the music, it as a catalyst to make creative enthusiasm, modal with what is done cannot be undone. But at the same time, some questions also emerged. One of the most significant that are some film music creation and researches out of the sounds the whole, that is to say, the film music is feasibility. Therefore, it is necessary in the creation and study of the film music, emphasizes from the angle of sound design, especially the film music creation process as an important issue to take into consideration.

The Music Software. Music making has been from the past mode of collective cooperation to personalization, personalized and economical small transformation. Combined with the latest digital multimedia technology of computer music production system, greatly reduce the cost of professional music production and recording, made it possible to low-cost, popular music production.

In music teaching and creation, using music software has become an important and necessary to study the content and research methods. (1) Digital music in its essence is a series of software data. From the point of view of informatics, these data must contain the able to comply with the principle of mathematical law of music. Because no one can out of it as a kind of mechanical nature of language, and mathematics principle is the principle of computer work. This makes music and the mathematics directly linked. (2) Sibelius as a music editor software, unlike the music editing software so widely application in the basic teaching area, but its unique plug-in ins function greatly expand the potential application of this software. Specially designed plug-in is Sibelius reserved a language for software programming module, and no limit function that can be used according to the user's demand to use software program design language internal procedures, to achieve the function of Sibelius itself does not have some. (3) The post-processing is a very important link of the recording. Cubase powerful effect is largely can replace the functionality of the hardware and on the handling of the sound, it can perform amplitude, time and echoes, such as the spectrum effect change, to change the tone, pitch and loudness of the sound properties. In the post-processing of music, Cubase with fully 
functional audio processing and MIDI record editing capabilities.

The Future of Computer Music. The production of electronic music equipment unit is composed of modules with different functions together. Mainly include: the voice module, processing module, editing, mixing and recording module and various modules together, in the form of components to complete a different job. Pure electronic music like the music, also want to use the recording tape in the process of production of cutting technology, the final result is recorded on the tape, so it is a period of specific music and pure electronic music known as "music tapes".

In the practical music, music is most obvious relationship with ideology and practical inherent in music, with its specific emotions and feelings to a certain social services. Especially the collective activities have played a role in strengthening the atmosphere that stimulates the collective mood, the combination of the social value of the music due to external factors into full play. More special is, because as a works or other social reasons under the condition of fixed, fixed repeated use, is people produce the conditioned reflex, into a signal has certain social significance [8].

Music is a kind of inner emotional expression as it is always something out of the sound structure of the external form. This stuff is mainly refers to human emotion, that is, the content of the music. It is the emotion and the content determines the sound structure of music works, overall development, determines the form of the music. Aesthetics is the study of each era characterized by the theory of the aesthetic consciousness of the form. Each the aesthetic consciousness of the times, always focus on the surface of each era now some great thinkers of aesthetic theory, and the great thinkers of the aesthetic theory is often embodied in a number of aesthetic category and aesthetic proposition.

\section{Conclusion}

In this paper, we conduct research on the development track of computer music and the influences on musical creation. Music as a special form of art, it is not a random sound stack, but rather "are grouped into specific culture acceptable mode type", is to give it a particular music culture types and specific forms, and the specific form is the rational framework that must be followed in the music creation. At the same time, the real emotion, rich imagination and inspiration of creation is also music culture to give specific types and specific forms of indispensable emotional elements. Therefore, the height of the rational and emotional harmony is the key to music creation. Under this background, we propose the computer music and the influences on musical creation topic that will enhance further advances of musical development that will be beneficial and meaningful.

\section{Reference}

[1] Stamatatos, Efstathios, and Gerhard Widmer. "Automatic identification of music performers with learning ensembles." Artificial Intelligence 165.1 (2005): 37-56.

[2] Castellano, Ginevra, et al. "Expressive control of music and visual media by full-body movement." Proceedings of the 7th international conference on New interfaces for musical expression. ACM, 2007.

[3] Zattra, Laura. "The Identity of the Work: agents and processes of electroacoustic music." Organised Sound 11.02 (2006): 113-118.

[4] Helen, Marko, and Tuomas Virtanen. "Separation of drums from polyphonic music using non-negative matrix factorization and support vector machine." Signal Processing Conference, 2005 13th European. IEEE, 2005. 
[5] Eigenfeldt, Arne, and Philippe Pasquier. "A realtime generative music system using autonomous melody, harmony, and rhythm agents." XIII Internationale Conference on Generative Arts, Milan, Italy. 2009.

[6] Sturm, Bob L., and Pardis Noorzad. "On automatic music genre recognition by sparse representation classification using auditory temporal modulations." Computer music modeling and retrieval (2012): 379-394.
[7] Lee, Eric, and Jan Borchers. "The role of time in engineering computer music systems." Proceedings of the 2005 conference on New interfaces for musical expression. National University of Singapore, 2005.

[8] Lee, Sang Won, and Jason Freeman. "echobo: A mobile music instrument designed for audience to play." Ann Arbor 1001 (2013): 48109-2121. 